\title{
Vitalija KAZLAUSKIENE
}

Université de Vilnius

vitalija.kazlauskiene@flf.vu.lt

http://orcid.org/0000-0003-0505-7770

\section{CATÉGORIE GRAMMATICALE DU SN : ANALYSE DU NOMBRE DANS LE CORPUS D'APPRENANTS LITUANIENS}

\section{INTRODUCTION}

Une simple liste des mots fréquemment utilisés par les apprenants ne fournit pas d'information suffisante sur leur compétence lexico-grammaticale, les mots n'étant, dans une langue donnée, jamais isolés. Pour ce qui est d'analyser la capacité des apprenants à employer les mots de façon cohérente dans un texte, il est plus approprié d'analyser des groupes de mots, des syntagmes et leurs composants. Pour les besoins de notre recherche empirique basée sur les données du corpus annoté des apprenants, nous avons choisi la catégorie grammaticale du nombre appliquée au syntagme nominal. En langue française, la catégorie morphologique du nombre se manifeste par des spécificateurs ou une altération des formes des lexèmes dans tout le complexe syntaxico-sémantique du syntagme et dépend directement de la forme du noyau (Zwicky 1993 : 298), présentant ainsi une certaine difficulté pour les apprenants lituaniens.

Nous avons analysé cette catégorie grammaticale dans les productions écrites des apprenants lituaniens en FLE à travers l'étude des difficultés rencontrées par ces derniers, des tendances générales de ses formes d'emploi en langue écrite (fréquence et ommission dans l'emploi des différents éléments) et de leur influence sur la qualité de la langue écrite même.

Dans cette optique, nous nous appuyons sur un certain nombre d'hypothèses récemment formulées quant à la structure du groupe no- 
minal. Il s'agira ici de présenter les résultats de l'analyse empirique du nombre au sein du syntagme nominal simple (déterminant + nom) dans l'examen final écrit des apprenants en FLE. Ces écrits dévoilent certaines tendances caractéristiques du niveau B1 quant à l'emploi du nombre dans le syntagme nominal.

\section{REMARQUES GÉNÉRALES SUR LA CATÉGORIE DE NOMBRE}

La catégorie de nombre est considérée comme l'opposition sémantique conceptuelle particulière entre l'unité et l'ensemble, entre unique et ordinaire, exceptionnel et commun, mais aussi comme l'opposition grammaticale entre singulier et pluriel. Le nom est de nombre singulier si la substance (état, chose) qu'il caractérise au moment de l'énonciation constitue une unité. Lorsqu'il s'agit de deux éléments ou plus, le nom est grammaticalement pluriel. La notion de nombre n'étant pas inhérente au nom, cette catégorie se rattache essentiellement au syntagme nominal. Le contexte, du discours et de la situation extralinguistique sont ainsi des éléments centraux pour l'accord en nombre. A ce titre, la quantification (ou catégorie de nombre) est interprétée comme une composante spécifique au groupe nominal (Cortès, Rousseau et Fourquet 1999 : 271).

La différence essentielle entre deux catégories aussi centrales que le genre et le nombre réside dans la possibilité de choix. En d'autres termes, le choix entre singulier et pluriel est quasiment toujours possible, ce qui n'est pas tout à fait le cas pour la catégorie du genre. La catégorie du nombre, en tant que catégorie morphologique, se manifeste à l'aide de spécificateurs ou d'altérations des formes des lexèmes qui dépendent de la forme du noyau. Par exemple, dans le syntagme nominal les belles robes, l'adjectif belles s'accorde au féminin pluriel du fait des particularités du nom robes. Ne portant pas eux-mêmes d'indices de genre et de nombre, l'adjectif comme le déterminant dépendent directement des caractéristiques du nom (Kazlauskienè 2017 : 150). Il faut cependant tenir compte du fait que ce sens peut parfois dépendre du contexte ou d'une situation particulière, étant donné que le sens grammatical est beaucoup plus abstrait que le sens lexical.

Dans les langues lituanienne et française, l'opposition des catégories singulier et pluriel est habituelle. Le point commun le plus manifeste entre les deux langues réside dans la présence de déterminants en français qui, 
pareillement aux suffixes en lituanien, marquent le sens grammatical. Partant de cette observation, on peut supposer que les apprenants lituaniens en FLE ne rencontreront pas de difficultés pour appréhender cette catégorie. Les données extraites du corpus nous permettent de mieux aborder cette hypothèse.

\section{PARTICULARITÉS DE LA CATÉGORIE DE NOMBRE DANS LE CORPUS DE LANGUE FRANÇAISE DES APPRENANTS LITUANIENS (RÉSULTATS DE L'ANALYSE EMPIRIQUE)}

\subsection{LE CORPUS}

Pour atteindre les objectifs établis en début d'article, les éléments suivants seront visés :

1. Constitution d'un corpus d'apprenants avec les compositions rédigées lors de l'examen final du FLE des écoles secondaires de Lituanie ;

2. Analyse des paramètres quantitatifs (listes de fréquence) et qualitatifs (concordances) du syntagme nominal dans le corpus d'apprenants ;

3. Analyse des particularités de la catégorie de nombre au sein du syntagme nominal dans le corpus étudié.

Le public cible est celui des élèves de la classe de terminale. Le nombre total de participants s'élève à 301. Pour l'année 2011 - 87 apprenants, $2012-67,2013-48,2014-47$ et 2015 - 52. L'âge moyen des élèves est de 18 ans $( \pm 1)$, et le niveau à atteindre est celui de B1-Utilisateur indépendant.

Dans cette analyse, deux parties de l'examen final ont été spécifiquement étudiées, à savoir celle des essais argumentatifs et celle des lettres. Tous ces travaux ont été introduits manuellement dans des fichiers électroniques; le corpus entier contient 102032 mots. Il est à noter que tous les textes analysés ont également été annotés manuellement. Cette annotation inclue le marquage de toutes les fautes et leur correction, ainsi que l'étiquetage de toutes les constructions possibles de SN, ce qui permet de distinguer plus précisément des formes de la catégorie de nombre et leurs particularités maîtrisées par les élèves.

Comme mentionné précédemment, le corpus se compose de textes écrits pendant l'examen final. La langue écrite implique une réflexion plus 
profonde et une planification de l'ordre plus poussée, ce qui permet de supposer que les élèves peuvent, en écrivant ces essais, produire la langue la plus correcte et performante possible au regard de leurs compétences et qu'ils visent à le faire. Tous les élèves ont traité le même sujet, et n'ont eu accès à aucun support supplémentaire. Par conséquent, le corpus utilisé pour les besoins de cette recherche peut être considéré comme la langue authentique des apprenants. L'observation de ces écrits nous a inspiré l'idée d'étudier la catégorie grammaticale du SN, et plus particulièrement celle de nombre. Dès lors, nous allons examiner et présenter les particularités de cette dernière dans le corpus de langue française écrite des apprenants lituaniens.

\subsection{LES PARTICULARITÉS DE LA CATÉGORIE DE NOMBRE DANS LE CORPUS}

\subsubsection{L'EXPRESSION DE LA CATÉGORIE DE NOMBRE DU NOYAU DE SN}

Dans la langue française, le déterminant (D) et le nom (N) sont les deux composantes principales du syntagme nominal et constituent la base de ce dernier. La catégorie grammaticale de nombre à l'intérieur du SN est toujours étroitement liée au sens du mot. Par exemple, certains mots français comme la pudeur, la gloire, l'innocence, la santé, le repos etc. ou certains mots lituaniens tels que grožis 'la beauté', kantrybe 'la patience', lygybe 'l'égalité', meile 'l'amour', šiluma 'la chaleur' etc. n'ont pas de pluriel car leur sens abstrait ne permet pas d'exprimer l'opposition d'une chose à plusieurs. Il en est de même pour le nom de certaines épices, matières (le genièvre, l'encens, l'or, l'argent), ainsi que les noms collectifs (la jeunesse), ou encore les noms propres (France, Lituanie). La base sémantique des noms indénombrables forme les noms des substances.

Il convient également de distinguer les noms qui, à la différence de ceux qui précèdent, n'ont pas de singulier. Ce cas se présente lorsque le mot exprime quelque chose de composé de plusieurs parties pouvant être interprétées comme une certaine accumulation quantitative (des $l u$ nettes, des mours, des frais etc.). En langue française comme en lituanien, certaines cérémonies sont toujours désignées au pluriel : les fiançailles, les funérailles, les noces etc.

On constate donc que la catégorie grammaticale de nombre en français et en lituanien présente des similitudes. On peut toutefois égale- 
ment noter des différences entre les deux langues. On trouve ainsi les mots dont le sens varie en fonction du nombre (un ciseau 'le ciseau de la censure' - des ciseaux 'instrument tranchant' ; un échec 'insuccès' - des échecs 'jeu de logique et de stratégie' ; une vacance 'poste vacant' - des vacances 'congé').

L'analyse empirique des données du corpus relève davantage de noms indénombrables (2 720 noms) que de noms toujours employés au pluriel (786 noms). Dans la plupart des cas (87\%), les apprenants appliquent correctement le nombre au nom. On observe des écarts lorsque le champ sémantique des deux langues, ici le français et le lituanien, ne coïncide pas. Le nom argent en est l'exemple le plus manifeste dans le corpus, dans la mesure où il est indénombrable en français mais pas en lituanien.

(1) Alors, $<X><O R D>$ \#la vie de nos jours\$ de nos $<$ SN8 $>$ jours $<X><R E D>$ \#la\$ notre $<$ SN0 $>$ vie $<L><C P V><E N G>$ \#devient\$ come très vite et nous devons savoir $<X><M A N>$ \#ce que $\$$ que nous allons faire, $<X><M A N>$ \#ce que\$ que nous allons manger, et où nous devons travailler pour gagner beaucoup $d^{\prime}<F><N B R>$ \#argent $\$<S N 8>$ argents, $<\ldots>$. (2011E301875)

(2) Il y a beaucoup de $<S N 8>$ médicaments et de tout pour aider les $<S N 0>$ gens, mais il faut avoir assez $d^{\prime}<G><N B R>$ \#argent $\$<S N 8>$ argents. (2011E301842)

Les exemples 1 et 2 illustrent l'emploi du mot argent au pluriel. On observe au total 29 emplois fautifs de ce mot dans le corpus. D'autres exemples montrent la position du nombre dans l'argumentation sémantique, qui est plus désignative en lituanien.

(3) Mais oui, il y a beaucoup de $<$ SN8 $><S N 3>$ problèmes pour moi: $<G><C O N J>$ \#en attendant\# atendent les $<S N 3>$ examens $d u<S N 8>$ français, $<G><N B R>$ \#des\$ de la $<G><N B R>$ \#mathématiques\$ $<$ SN8> mathématique, de la $<$ SN8> biologie... (2015L2643D68)

(4) Mon $<$ SN2 $>$ professeur préféré est $<X><M A N>$ \#celui $\$<G><N B R>$ \#des mathématiques\$ de la $<$ SN8> mathématique. (2014Lpra5)

Dans les deux exemples ci-dessus, les apprenants lituaniens choisissent le singulier pour la discipline des mathématiques au lieu de la forme plurielle du nom, qui en français est considéré comme le nom collectif 
comprenant l'ensemble des six branches des mathématiques, à savoir l'algèbre, l'analyse, la géométrie, l'arithmétique, le calcul, la mécanique. Les exemples 1 à 4 montrent une forte influence des différences langagières sur les productions écrites et comme résultat - l'interférence du lituanien sur le français.

La langue maternelle (dans le cas analysé - le lituanien) ne prédétermine toutefois pas à elle seule l'application fautive du nombre à un nom donné. On trouve ainsi dans le corpus le nom politics au lieu de la forme française politique, ce qui laisse plutôt penser à une influence de la langue anglaise, laquelle est dans la plupart des cas la première langue étrangère des apprenants lituaniens. Les erreurs de nombre peuvent également s'expliquer par l'intuition linguistique personnelle des apprenants, comme le montrent les exemples suivants :

(5) Si nous voulons rester $<G><P R E><M A N>$ \#en\$ $<G><G E N>$ \#bonne\$ bon $<S N 1>$ forme il faut manger beaucoup de $<S N 8>$ fruits et $<G><P R E><M A N>$ \#de\$ $<F><D I A>$ \#légumes\# legumes et il ne faut pas manger $<L><$ SIG $>$ \#fastfood $\$$ les vite $<M><M C O>$ \#nourriture\$ $<$ SN1 $>$ nuritures ce n'est pas bon pour notre bonne $<$ SN1 $>$ forme. (2013E165425)

(6) Comment tu $<L><$ SIG $>$ \#passe\$ fais $<G><$ PER $><G><N M>$ \#tes\$ sa $<G><$ NMR $>$ \#tes\# $<$ SN0 $>$ vacance? (2011L3018)

(7) Quoi qu'il en soit, je veux étudier $<G><P R E>$ \#à\$ dans $l^{\prime}<S N 3>$ université de $<$ SN8 $><N>$ Gediminas, parce que j'aime $<G><N B R>$ \#les\$ la $<F><D I A>$ \#médias\$ $<$ SN0 $>$ media et les $<F><D I A>$ \#technologies\$<SN2> téchnologies modernes. (2015L2643D10)

L'exemple 6 correspond à ce que nous avons observé précédemment dans le corpus, lorsque le sens même du nom dépend du nombre employé et qu'un emploi fautif du nombre altère le sens du mot. Dans cet exemple, c'est le nom vacance(s) qui est employé incorrectement. L'exemple 7 expose quant à lui la difficulté rencontrée face à la différence d'emploi entre le français et la langue anglaise pour l'expression les médias (mass media). Cet exemple ne constitue pas l'unique cas où l'on peut discerner l'influence de la langue anglaise sur les écrits en français. L'exemple 8 ci-dessous applique la règle de la dérivation qui correspond à une règle en langue anglaise par laquelle le singulier en $-y$ devient -ies au pluriel. 
(8) D'autre $<$ SN8 $>$ coté il faut donner beaucoup de $<S N 8><S N 3><S N 4>$ liberté aux $<$ SN8 $>$ jeunes pour choisir leur $<M><M C O>$ \$hobbys\$ $<$ SN0> hobbies. (2015E2643D26)

D'autres fautes portant sur la catégorie grammaticale de nombre sont plutôt de source morphologique. Il est à noter qu'en français, le pluriel d'un nom est le plus souvent marqué uniquement à l'écrit (un oiseau/des oiseaux, un livre/des livres). On trouve parfois une altération de la dernière syllabe (un cheval/des chevaux). Les exemples observés dans notre corpus peuvent laisser supposer que la faible présence de marquage du pluriel à l'oral en français pourrait être la cause des formes fautives à l'écrit pour les apprenants. En voici quelques exemples :

(9) J'ai beaucoup de $<G><N B R>$ \#plans\$ $<L><L T>$ \#projets $\$<S N 3>$ plan pour $l^{\prime}<$ SN8 $>$ avenir. (2015L2643D21)

(10) En plus, on pourrait $d<G><P R E><R E D>$ \#nereikia de\$ de continuer notre $<$ SN2 $>$ vie musicale, on pourra chanter dans $\langle G><N B R>$ \#tous\$ tout les $<M><M C O>$ \#festivals\$ $<$ SN3 $>$ festivale à $<S N 8><N>$ Vilnius. (2015L2642D90)

(11) Grâce d̀ $<$ SN8 $>$ Internet nous savons plus que $<G><A R T><M A N>$ \#des\$ $<$ SN3 $>\quad<M><C O>$ \$encyclopédistes\$ encyclopediste de XVIIIieme $<$ SN02> siecle. (2015E2643D33)

(12) Encore, il y a beaucoup de $<S N 1.4><S N 3.1>$ personne différentes dans le $<$ SN1.4> monde entier, <...> (2015E2643D25)

Les exemples 9 à 12 ne marquent pas le pluriel et ses traits qui sont indispensables compte tenu du champ lexical et du contexte. En opposition à la présence du quantificateur beaucoup, les apprenants donnent la préférence à la forme singulière pour le nom. En dehors de ces phrases singulières $(0,3 \%$ des cas), on constate que la forme plurielle des noms dans le corpus correspond à la situation contextuelle.

On distingue en français trois formes graphiques particulières au pluriel : $-s,-x$ et morphème zéro. Le morphème dominant est celui du $-s$. Selon New (2004), 98\% des formes plurielles des noms sont marquées par un $-s$, qui est en même temps morphème productif et régulier. Le morphème $-x$ apparaît quant à lui beaucoup plus rarement. On peut donc supposer que cette prédominance de la forme -s conduit les apprenants à s'habituer à noter le pluriel à l'aide du $-s$ plutôt que du $-x$. 
(13) $A u<$ SN8 $>$ premier, la $<$ SN3 $>$ plupart des $<$ SN8 $><S N 3>$ jeunes de 14-18<SN02 $>$ ans (à peu près) pensent, qu'ils sont les $<M><M C O>$ \#dieux $\$<$ SN0> dieus: ils peuvent faire n'importe quoi. (2015E2643D68)

(14) Je voudrais $<G><P R E><R E D>$ \#nereikia $d \$ d^{\prime}<M><M C O>$ \$inviter\$ iviter $<X><O R D>$ \#t'inviter\$ toi $<G><P R E><R E D>$ \#nereikia pour\$ pour créer $l^{\prime}<L><E N G>$ \#agence $\$<S N 3>$ agency $<G><N B R>$ \#des\$ de $<G><N B R>$ \$travaux\$ $<$ SN1.4 $>$ travails audiovisuels. (2015L2643D34)

(15) Cependant certains $<M><M C O>$ \#choix $\$<$ SN3 $>$ choixs comme $<L>$ $<C P N>$ \#alcool\$ l' $<$ SNO $>$ ahlool(?), les $<$ SNO $>$ drogues ou même les $<$ SN2 $>$ réseaux sociaux peuvent avoir beaucoup de $<$ SN1.5> conséquences $<X><O R D>$ \#mauvaises conséquences\$ mauvaises. (2015E2643D50)

Les phrases 13 à 15 sont des exemples ostensibles de la préférence des apprenants pour la forme en $-s$ pour les noms au pluriel. Ce morphème est employé au lieu du - $x$ (ex. 13), et ce même en présence de l'opposition -ail/-aux (ex. 14). On l'observe même couplé au morphème zéro (ex. 15), lequel assure les mêmes fonctions qu'un morphème et se distingue à sa propre manière par son sens dérivationnel ou relationnel.

Le morphème - $x$ est en effet beaucoup plus rare en français que le -s. Il est intrinsèque aux noms portant les flexions -eu et $-a u$, parfois -ou. Les noms qui s'écrivent au pluriel avec $-x$ présentent des caractéristiques différentes, les suivantes étant les plus importantes :

- La formation morphologique de certains noms en $-x$ au pluriel est identique à ceux en $-s$, c.à.d. que l'on ajoute à la racine d'un mot un suffixe phonétiquement non-marqué (bateau - bateaux). Dans d'autres cas, la forme du suffixe change, et il est fortement marqué phonétiquement (animal - animaux). Dans cette situation, la majorité des occurrences fautives correspond aux noms avec la flexion muette, comme on peut l'apercevoir dans les exemples 16 et 17 ; au lieu du $-x$, les apprenants emploient le -s. Dans le cas du pluriel marqué phonétiquement, le nombre de fautes est plus faible (ex. 18 et 19).

(16) J'aime $<F><D I A>$ \#recevoir\$ reçevoir beaucoup de $<G><N B R>$ $<F><G R A>$ \#cadeaux $\$<$ SN8 $>$ cadea $\hat{s}$ et être avec mes $<$ SN8 $>$ amis. (2013L165425) 
(17) $<F><G R A>$ \#puis\$ Pui, j'ai beaucoup $d^{\prime}<$ SNO $>$ activités comme jouer $<G><P R E>$ \#au\$ le $<$ SN8 $>$ volleyball $<\ldots>$, visiter les $<$ SN0 $>$ musées et les $<$ SN2 $><G><N B R>$ \#lieux\$ lieus inconnus. (2011L301844)

(18) Par $<M><M C O>$ \#exemple $<$ SN8 $>$ excemple lire des $\langle G><N B R>$ \#journaux $\$<S N 0>$ journals, lire des $<G><A R T><R E D>$ \#nereikia $d^{\prime} \$ d^{\prime}<G><N B R>$ \#histoires\$ $<$ SNO $>$ histoire. (2011E301898)

$<\ldots><G><A R T><M A N>\# l \$<S N 0>$ Internet $<G><P R N>$ \#nous\$ notre $<L><C P V>$ \#donnent\$ doivent beaucoup $<G><P R E>$ \#de\$ des $<M><M C O>$ \#possibilités $\$<S N 4>$ posibilites pour communiquer et aident $<G><P R E><M A N>\#$ à faire des $<G><N B R>$ \$travaux $\$$ $<$ SNO $>$ travailes plus vite. (2013E165470)

- Le cas où le nom apparaît dans la langue plus souvent au pluriel qu'au singulier (œil - yeux) ou au contraire plus souvent au singulier qu'au pluriel (drapeau - drapeaux). Avec $0.13 \%$ des mots exprimés, ce type de noms avec la dimension $-x$ ne sont pas suffisamment présents dans le corpus pour permettre de tirer des conclusions particulières.

Il est à noter que les élèves font non seulement l'erreur d'employer le $-s$ au lieu du $-x$, mais également la faute inverse. Ces erreurs semblent liées au système même de la langue française, basé sur l'exception, comme le pluriel des noms en -ou qui se forme par l'ajout du $-s$, à l'exception de quelques noms qui au pluriel prennent un $-x$, tels que chou, genou, caillou, bijou, pou, hibou, joujou. Cette règle d'exception est parfois appliquée à d'autres noms qui au singulier se terminent par -ou, par exemple :

(20) $<$ SN0 $><G><N B R>$ \#bisous\$ Bisoux, $<N>$ Natalie (2011L301853)

En langue française la catégorie morphologique de nombre est exprimée seulement au pluriel à l'exception de quelques cas comme animal - animaux, corail - coraux. Par conséquent, la forme singulière du nom n'est pas suffisante pour avoir l'information complète sur la compétence grammaticale des apprenants. Cette observation justifie la fréquence de l'analyse de l'expression du pluriel du SN dans ce travail.

En dépit des difficultés mentionnées précédemment, on constate que les apprenants appréhendent et considèrent la catégorie de nombre des noms. Cette catégorie étant présente dans la langue maternelle (lituanien) et dans la première langue (celle de l'anglais), ceci n'est pas étonnant. La deuxième explication de ces résultats pourrait être que le nom est l'un 
des éléments que l'on appréhende très tôt dans l'apprentissage d'une langue. D'après Bartning (1997), deux tiers des mots que la plupart des apprenants intègrent le mieux à l'école sont justement les noms et les numéraux.

En ce qui concerne l'accord à l'intérieur même du syntagme nominal, la situation est beaucoup plus compliquée. Si le noyau nominal possède les traits du pluriel, ces traits devraient être également reflétés dans les autres composantes du SN, telles que le spécificateur et l'adjectif. Les limites de ce travail ne nous permettent pas d'analyser l'expansion complète du pluriel dans le $\mathrm{SN}$ à droite et à gauche. Comme il a été noté en début d'article, nous avons limité le champ d'analyse de cette étude aux éléments structuraux principaux du SN tels que le spécificateur et le nom lui-même, pour lequel la catégorie de nombre dans le corpus analysé est présentée ci-avant. La partie suivante porte sur les particularités du spécificateur dans le corpus d'apprenants.

\subsubsection{L'EXPRESSION DE LA CATÉGORIE DE NOMBRE DES SPÉCIFICATEURS DE SN}

Le terme spécificateur signifie «le mot qui définit». Dans la grammaire générative d'aujourd'hui, la principale condition d'être un spécificateur est formelle, c'est-à-dire qu'il se présente en tant que mécanisme de vérification des indices formels (ceux de genre, nombre, personne, etc.) en envisageant l'accord entre les indices de constituant principal du syntagme et ceux du spécificateur. Dans le domaine nominal, le spécificateur se présente sous forme de déterminant, de quantificateur et d'adjectif. Les déterminants méritent une mention particulière dans la mesure où ils ne sont pas seulement les morphèmes qui représentent les catégories grammaticales du nom, mais également un moyen supplémentaire de nuancer le sens, la jonction du thème au rhème, l'expression de l'intertextualité dans le cadre du texte dans son ensemble ou d'une seule énonciation. Les déterminants sont le moyen principal de coordination de la référence (Spraunienè 2008 : 109 ; Kazlauskienè 2016 : 60). Leur choix dépend du sens pragmatique du texte dans son ensemble.

Dans le cadre de cet article, nous portons une attention particulière aux propriétés des spécificateurs (notamment celles des déterminants) pour transmettre les indices du nombre des noms, autrement dit aux particularités des spécificateurs dans la formation du sens quantitatif des syntagmes nominaux. En langue française, le vecteur d'expansion 
de la catégorie de nombre est orienté aussi bien à droite qu'à gauche. Les spécificateurs (déterminants et quantificateurs) du syntagme nominal français se posent toujours en présupposition de ce dernier. Ils sont réflecteurs sémantiques et morphologiques de la catégorie grammaticale, et dans ce cas de celle de nombre. Le tableau 1 représente tous les spécificateurs qui expriment le pluriel dans le corpus analysé.

TABLEAU 1: La liste des spécificateurs du pluriel du corpus

\begin{tabular}{|c|c|}
\hline Spécificateur à gauche & $\mathrm{n}$ \\
\hline \multicolumn{2}{|c|}{ Article pluriel } \\
\hline Les & 5376 \\
\hline Des & 2440 \\
\hline \multicolumn{2}{|c|}{ Adjectifs possessifs au pluriel } \\
\hline Mes & 686 \\
\hline Tes & 112 \\
\hline Ses & 206 \\
\hline Nos & 376 \\
\hline Leurs & 312 \\
\hline \multicolumn{2}{|c|}{ Adjectifs démonstratifs au pluriel } \\
\hline Ces & 225 \\
\hline \multicolumn{2}{|c|}{ Quantificateur adverbial } \\
\hline Beaucoup de & 1282 \\
\hline Peu de & 76 \\
\hline Trop de & 53 \\
\hline Plus de & 708 \\
\hline Assez de & 57 \\
\hline Tant de & 6 \\
\hline \multicolumn{2}{|c|}{ Quantificateur nominal } \\
\hline La plupart de & 40 \\
\hline La majorité de & 46 \\
\hline Une partie de & 55 \\
\hline Une somme de & 12 \\
\hline Un groupe de & 12 \\
\hline Une variété de & 12 \\
\hline Un type de & 11 \\
\hline Une quantité de & 7 \\
\hline \multicolumn{2}{|c|}{ Autres quantificateurs } \\
\hline Plusieurs & 40 \\
\hline Chaque & 371 \\
\hline Déterminant numéral cardinal & 398 \\
\hline Quelques & 130 \\
\hline Tous (-tes) & $447(156)$ \\
\hline Certain(e)s & 58 \\
\hline Autres & 18 \\
\hline Plein de & 64 \\
\hline
\end{tabular}


Les données présentées dans le tableau ci-dessus reflètent les moyens privilégiés par les élèves pour exprimer le pluriel du nom, ce qui pourrait traduire les préférences des apprenants caractéristiques au niveau à atteindre B1, concernant le choix d'un spécificateur. Comme on peut l'observer dans le tableau 1, le spécificateur le plus répandu est l'article (les, des). Parmi les quantificateurs, le spécificateur le plus utilisé pour exprimer une quantité approximative est beaucoup de.

Les données empiriques n'exposent pas ou peu certains autres moyens pour marquer le pluriel. Par exemple, le corpus analysé n'est pas représentatif en ce qui concerne le groupe particulier des spécificateurs formés à partir des mots relationnels tels qu'un litre, un verre, un pot, une barquette, etc., lesquels fonctionnent comme des spécificateurs en français et exigent d'être accompagnés par des noms de contenu sémantique différentiel, par ex : un litre de lait, un verre de vin, etc. Ces résultats s'opposent partiellement à ceux de Parodi, Schwartz et Clahsen (1997) qui, par l'étude de l'allemand L2, prouvent que les apprenants de niveau assez bas utilisent d'abord les moyens lexicaux pour marquer le pluriel. Le corpus analysé, comme le montre le tableau 1, est quant à lui rempli de quantificateurs lexicaux, mais aussi de différents déterminants numéraux cardinaux. Les déterminants restent la forme plurielle la plus répandue.

Nous nous sommes aperçue que les sujets des travaux écrits ont une influence directe sur l'inventaire lexical utilisé par les élèves. Nous présupposons que c'est la raison pour laquelle on constate cette différence quantitative considérable entre les formes plurielles des déterminants et quantificateurs logiques (Dominicy 1984 : 167), autrement dit quantificateurs conventionnels (Mikulskas 2009) : tout, tous les, quelque(s), nul, aucun. Le niveau langagier des apprenants se reflète non seulement dans leur compétence grammaticale, mais aussi dans leur habitude d'exprimer une idée à l'aide de constructions acquises telles que beaucoup de, plus de, une partie de, etc. Dans son ensemble, le corpus relève nettement les préférences des élèves. En règle générale, les apprenants n'ont pas recours à des formes ou constructions particulières pour exprimer les nuances de pluralité. Les spécificateurs sont assez simples, élémentaires, et souvent répétés dans la production écrite.

Pour ce qui est des difficultés liées aux spécificateurs du pluriel, nous remarquons tout d'abord que la forme auditive des déterminants pluriels influence l'emploi plus au moins correct à l'écrit dans le cas des syllabes 
et lettres prononcées, et au contraire, dans le cas où elles sont muettes les fautes sont plus répétitives. Nous pouvons entrevoir ce phénomène dans les exemples suivants :

(21) $<\ldots>$ il y a un $<$ SN6 $>$ article qui $<F><D I A>$ \#présente\$ prèsente les $<S N 3>$ études sur les $<$ SN8> jeunes: la $<$ SN3> majorité $d^{\prime}<M>$ $<M C O>$ \#adolescents\$ $<$ SN11 $>$ adolicents, qui n'ont pas assez de $<$ SN8 $>$ liberté, a $<G><A R T><M A N>\# l a \$<S N 4>$ possibilité de développer la $<F><D I A>$ \#dépression $\$<S N 0>$ depression.

(2015E2643D40)

(22) Alors, les $<M><M C O>$ \#parents $\$<S N 0>$ parners ne doivent pas s'occuper de leurs $<S N 8>$ enfants toujours, si non, les $<S N 23>$ enfants ne $<G><C O N J>$ \#seront\$ seraienet prêts à vivre seuls.

(2015E2643D60)

Ces exemples montrent une corrélation nette entre graphème et phonème. Nous nous sommes aperçue que même la forme de l'adjectif possessif leurs, dont la prononciation ne se distingue pas de celle de la forme singulière leur, est correcte dans le cas de la présence vocalique d'une liaison. La situation est similaire pour l'adjectif démonstratif ces, qui parfois apparaît dans le corpus au lieu de la forme homophone ses, par exemple :

(23) Notre $<$ SNO $>$ corps $<L><S I G>$ \#a\$ est $<$ SN3 $>$ besoin $<G><A R T>$ \#des\$ de $<$ SN8 $>$ légumes et $<G><A R T>$ \#des\$ $<$ SNO $>$ fruits mais on ne mange assez de $<F><H O M>$ \#ces\$ ses $<$ SN8 $>$ produits. (2013E165412)

La différence entre les systèmes des langues lituanienne et française alimente également la présence d'occurrences fautives ; pour l'ensemble des 93 cas de ses observés dans le corpus, 46 sont erronés. Les élèves donnent la priorité au possessif ses au lieu de leurs prévu par le contexte.

(24) À cause de $l^{\prime}<S N 8>$ Internet les $<$ SNO $>$ parents perdent les $<S N 3>$ relations avec $<G><P R N>$ \#leurs $\$$ ses $<S N 8>$ enfants. (2014Epra12)

(25) Ils ne font pas $<G><P R N>$ \#leurs $\$$ ses $<S N 0>$ devoirs parce que c'est rien, ils ont $<G><A R T>$ \#de la\$ la $<$ SNO $>$ liberté. (2015E2642D76) 
(26) Avec la $<$ SN1.4 $>$ liberté $<F><D I A>$ \#nécessaire\$ necessaire ils n'ont pas de $<S N 8><S N 3>$ questions comment ils doivent commencer $<G><P R N>$ \#leurs\$ ses $<$ SN3 $>$ vies sans la $<$ SN8 $><$ SN6 $>$ famille qui était avec eux tout ce $<S N 1>$ temps. (2015E2643D46)

Dans ces exemples, nous pouvons distinguer l'influence de la langue lituanienne dans laquelle, tout comme en anglais, les adjectifs possessifs s'accordent seulement avec le possesseur indiquant le nombre et non l'objet possédé. Ce n'est pas le cas en français où le possessif, comme cela a déjà été évoqué précédemment, s'accorde aussi bien avec le possesseur qu'avec l'objet possédé. Il est à noter que, sans compter les cas erronés présentés ci-dessus, les déterminants utilisés par les élèves dans le corpus sont en général corrects.

Concernant les quantificateurs adverbiaux, dont le nombre d'occurrences dans le corpus est assez faible (cf. tableau 1), nous avons constaté des erreurs au sein même des constructions bien connues des élèves. Comme le démontrent les exemples suivants, l'emploi de la préposition de dans ce type de constructions de quantificateurs, comme : beaucoup, peu, trop, plus, assez, tant etc., est souvent en question.

(27) C'est la $<S N 6>$ fête quand $<G><G E N>$ \#toute\$ tout $<G><N B R>$ \#la\$ les $<F><G R A>$ \#famille\$ familes $<G><V P R N>$ \#se\$ rencontre chez leur $<$ SN8 $>$ parents, quand il y a beaucoup $<G><P R E>\#$ de $\$$ des $<$ SN3.2 $>$ plats sur la $<$ SN8 $>$ tabale et aussi quand $t u<G><C O N J>$ \#peux\$ peut avoir beaucoup $<G><P R E>$ \#de\$ des $<$ SN8 $>$ cadeaux. (2013L165429)

(28) $<\ldots><X><R E D><G><P R E><R E D>$ \#nereikia dans\$ dans $<G>$ $<G E N>$ \#ce\$ cette $<$ SN8 $>$ jour il peut manger beaucoup $<G><P R E>$ $\#$ \#de\$ des $<$ SN8 $>$ bonbons et une $<S N 9>$ tarte citron $<Q><T R O>$, $<\mathrm{X}><\mathrm{COH}>$ \#que\$ quel notre $<\mathrm{SN} 0>$ mère $<\mathrm{G}><\mathrm{CONJ}>$ \#prépare $\$$ prépares chaque $<$ SNO $>$ année. (2013L165436)

(29) Beaucoup $<G><P R E>\# d^{\prime} \$$ des $<S N 8>$ enfants $<G><N B R>$ $<$ PER $>$ \#prennent\$ prends $<G><A R T>$ \#des\$ les $<L><E N G>$ \#exemples\$ $<S N 3>$ examples $<G><P R E>$ \#de la\$ pour violence et $<G><A R T><M A N>$ \#des\$ $<$ SN3 $>$ choses comme ça $<F><H O M>$ \#sur\$ sûr la $<$ SN8> télé. (2011E301876)

Dans le cas des quantificateurs nominaux, on observe soit l'omission de l'article (ex. 30, 31), soit son emploi erroné (ex. 32). 
(30) Aujourd'hui $<G><A R T><M A N>$ \#la\$ plupart $<G><A R T>$ \#des\$ les $<$ SN8 $>$ gens ne $<G><N B R>$ \#savent\$ sait pas $<L><S I G>$ \#comment $\$$ comme habiter sans les $<S N 2>$ technologies modernes. (2011E301859)

(31) Les $<$ SN2 $>$ technologies modernes sont importantes pour $\langle G\rangle\langle A R T\rangle$ \#la $\$$ plupart des $<$ SN8 $>$ personnes. (2011E301929)

(32) Dans notre $<S N 8>$ jours $<G><G E N>$ \#la $\$$ le $<S N 3>$ plupart des $<S N 8>$ gens discutent $<F><G R A>$ \#nereikia $-\$$ que-ce-que nous devons faire pour rester en bonne $<$ SN1> forme? (2013E165457)

$<\ldots><G><N B R>$ \#ce sont\$ c'est les $<$ SN3 $>$ technologies modernes pour nous, mais $<Y><L O U>$ il n'y a pas modernes pour $<G>$ $<$ ART $><$ MAN $>$ \#une $\$ G><$ NBR $>$ \#somme $<<$ SNO $>$ sommes $<G>$ $<$ PRE $><M A N>$ \#de\$ $<M><M C O>$ \#personnes\$ $<$ SN6 $>$ persones $<Q><$ TRO $>$ \#,\$, qui $<G><P R E><R E D>$ \#nereikia à $\$ \grave{a}<G><N B R>$ \#travaillent\$ travaille avec $<G><A R T><M A N>$ \#les\$ $<$ SN8 $>$ technologies, <...> (2011E301931)

Les apprenants de ce niveau connaissent souvent bien les marques d'opposition grammaticale et leur nécessité à l'intérieur du syntagme nominal, mais ne les appliquent pas. Les stratégies compensatoires se présentent soit sous des formes simplifiées, soit dans l'omission des traits de l'accord. Les exemples (34-36) montrent une tendance des élèves à exprimer le pluriel seulement à l'aide des spécificateurs sans accord du nom. Ceci ne se répète pas pour les syntagmes nominaux avec déterminants en tête.

(34) En plus, on pourrait $d<G><P R E><R E D>$ \#nereikia de\$ de continuer notre $<S N 2>$ vie musicale, on pourra chanter dans tout les $<M><M C O>$ \#festivals\$ $<$ SN3 $>$ festivale à $<$ SN8 $><N>$ Vilnius. (2015L2642D90)

(35) J'ai beaucoup de $<G><N B R>$ \#plans\$ $<L><L T>$ \#projets\$ $<$ SN3 $>$ plan pour $l^{\prime}<$ SN8 $>$ avenir. (2015L2643D21)

(36) Encore, il y a beaucoup de $<S N 1.4><S N 3.1>$ personne différentes dans le $<$ SN1.4 $>$ monde entier, et $<G><G E N>$ \#le $\$$ la $<S N 0>$ gouvernement ne $<G><N B R>$ \#doit\$ doivent pas prendre $<G><A R T>$ \#des\$ les $<$ SN3 $>$ lois pour toutes les $<S N 8>$ personnes, mais par $<S N 8>$ exemple seulement les $<$ SNO> jeunes. (2015E2643D25) 
Pour généraliser les résultats de ces données, il ressort clairement du corpus annoté que les apprenants lituaniens visant le niveau B1 en français et n'ayant pas encore ni une connaissance intuitive de la grammaire, ni les habitudes de bien l'appliquer, ont finalement conscience de leur aptitude morphosyntaxique. $87 \%$ des particularités de la catégorie de nombre sont en effet bien respectées.

\section{CONCLUSIONS}

Notre analyse nous amène à formuler les conclusions suivantes :

1. Premièrement, même les données d'un corpus d'ampleur modeste permettent de distinguer assez nettement que les apprenants du niveau analysé savent bien qu'il faut indiquer l'opposition grammaticale. La forme du nombre est morphologiquement bien appliquée au sein du SN dans $87 \%$ des cas.

2. Dans son ensemble, le corpus revèle nettement les préférences des élèves. Les données montrent le manque d'inventivité des apprenants en matière d'expression du nombre : ils ne varient pas les spécificateurs, et choisissent le plus souvent d'utiliser les moyens de distinction, de marquage et de précision assez simples et répétitifs. L'article défini compte parmi les spécificateurs les plus répandus à gauche.

3. En ce qui concerne la catégorie de nombre, les apprenants se trompent parfois en attribuant au collecteur de l'accord un indice de nombre sans prêter attention à la position du détecteur de l'accord. Nonobstant, les fautes d'accord des déterminants sont insignifiantes.

4. Les ressemblances entre éléments linguistiques de différentes langues sont souvent la cause d'interférence. On discerne l'influence de la langue lituanienne aussi bien que celle de la langue anglaise dans les productions écrites des apprenants. Les interférences morphologiques du nombre sont présentes en quantité non-négligeable.

5. En plus de ces erreurs interlinguales, on remarque également la présence de fautes intralinguales, à savoir une corrélation nette entre graphème et phonème.

\section{BIBLIOGRAPHIE}

Bartning I., 1997, "L'apprenant dit avancé et son acquisition d'une langue étrangère, acquisition et interaction en langue étrangère », Acquisition et interaction en langue étrangère 9, pp. 9-50. 
Cortès C., Rousseau A., Fourquet J., ed. 1999, Catégories et connexions : en hommage à Jean Fourquet pour son centième anniversaire le 23 juin 1999, Linguistique, Villeneuve d'Ascq, Presses universitaires du Septentrion.

Dominicy M., 1984, La naissance de la grammaire moderne : langage, logique et philosophie à Port-Royal. Philosophie et langage, Bruxelles, P. Mardaga.

Kazlauskiene V., 2016, « Daiktavardinio junginio gramatinio apibrèžtumo raiška lietuvių, besimokančių prancūzų kalbos, tekstyne », Santalka: filologija, edukologija 24 (1), pp. 55-64.

Kazlauskienè V., 2017, «Prancūzų K2 daiktavardinio junginio giminès gramatinès kategorijos kompetencija », Taikomoji kalbotyra 9, pp. 149-175, www.taiko mojikalbotyra.lt, consulté le 14.01.2018.

Mikulskas R., 2009, "Jungties konstrukcijos ir jų gramatinis kontekstas », Acta Linguistica Lithuanica LXI, pp. 113-156.

New B., 2004, "Le traitement des mots singuliers et pluriels en français et en anglais », Fondation Fyssen 19, pp. 131-138.

Parodi T., Schwartz B.D., Clahsen N., 1997, " On the L2 acquisition of the morphosyntax of German nominals », Essex research reports in linguistics 15, pp. 1-43.

Spraunienè B., 2008, «Paprastųjų ir įvardžiuotinių būdvardžių opozicija lietuvių kalboje kaip apibrěžtumo sistema », Vilniaus Universitetas, Acta Linguistica Lithuanica LIX, pp. 109-139.

Zwicky A.M., 1993, «Heads, bases and functors », in: Corbett G.G., Fraser N.M. \& McGlashan S. (eds), Heads in grammatical theory, Cambridge, Cambridge University Press, pp. 292-315.

\section{CATÉGORIE GRAMMATICALE DU SN : ANALYSE DU NOMBRE DANS LE CORPUS D'APPRENANTS LITUANIENS}

\section{Résumé}

En langue française, la catégorie morphologique du nombre se manifeste par des spécificateurs ou une altération des formes des lexèmes dans tout le syntagme nominal, présentant ainsi une certaine difficulté pour les apprenants lituaniens.

Nous avons analysé cette catégorie grammaticale dans les productions écrites des apprenants lituaniens en FLE à travers l'étude des difficultés rencontrées par ces derniers, des tendances générales de ses formes d'emploi en langue écrite (fréquence et ommission dans l'emploi des différents éléments) et de leur influence sur la qualité de la langue écrite même.

Mots-clés : catégorie grammaticale, syntagme nominal, nombre, corpus d'apprenants lituaniens 
GRAMMATICAL CATEGORY OF THE SN: ANALYSIS OF THE NUMBER IN CORPUS OF LITHUANIAN LEARNERS

\section{Summary}

In French, the morphological category of the number is manifested by specifiers or an alteration of the forms of lexemes throughout the nominal phrase, thus presenting a certain difficulty for Lithuanian learners.

We have analyzed this grammatical category in the written productions of Lithuanian learners in FLE through the study of the difficulties encountered by them, general tendencies of their forms of employment in written language (frequency and omission in the employment of the different elements) and their influence on the quality of the written language itself.

Key words: grammatical category, noun phrase, number, corpus of Lithuanian learners 